\title{
Flare Rate Spectra as a Possibility of Diagnostics of Convection Zones in Stars
}

\author{
L.A. Pustil'nik \\ Special Astrophysical Observatory, \\ USSR Academy of Sciences, N.Arkhyz, 357147, USSR
}

\begin{abstract}
A theoretical interpretation is offered for the power-law dependence of the flare occurrence rate in red flare dwarfs. Flares on red dwarfs are regarded as being physically akin to solar flares, with the energy source being the magnetic field. A power law flare energy relation then emerges naturally. Model estimates of the corresponding spectral index $\beta$ fit the observed values in most flare stars. Within the bounds of the interpretation offered the energy dependence of a flare event is determined by the character of the turbulence in the convection zone. The observed parameters of the dependence can be used to diagnose the character of the turbulence in the convection zones of flare stars.-
\end{abstract}

\section{The physical nature of the flare rate spectrum}

Early investigations of flare activity in stars addressed the problem of how the flare rate depends on the emitted flare energy (Gershberg, 1972; Lacy et al., 1976). The flare rate spectrum was defined as the number of flares with energy greater than a threshold energy $E_{0}$, such that $\nu\left(E>E_{0}\right) \propto E_{0}^{\beta}$.

A theoretical analysis (Pustil'nik, 1988) has shown that the observed universality can be explained as a manifestation of the dissipation of magnetic fields in stellar atmospheres (Gershberg and Pikel'ner, 1972). The initial magnetic fields are considered to be the result of dynamo processes. The magnetic structures are expelled from the convection zone by turbulent motions and magnetic buoyancy. Physically the approach is rather transparent since the flare energy and the rate depend much on the same parameter, namely a dimension $L$ of the magnetic structure. The flare energy is given by

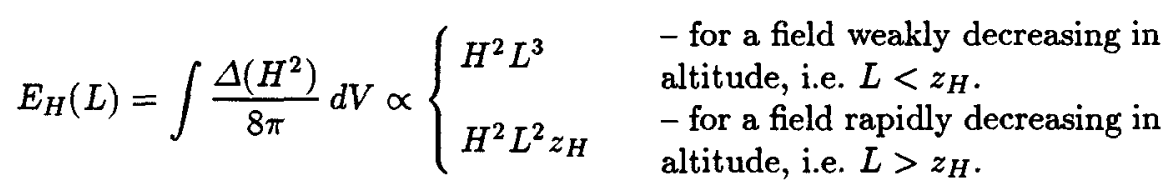


On the other hand the rate of occurrence of flares is determined both by the number of such structures on the star disk $N_{s t r} \propto R_{\star}^{2} / L^{2}$ and by their lifetime $\tau(L) \cong L / V(L)$. Here $R_{\star}$ is the dimension of of the star and $V(L)$ is a turbulent velocity in the corresponding turbulent cell of dimension $L . z_{H}$ is the lengthscale of magnetic field. $V(L)$ is determined by a specified turbulence spectrum for the convection zone of the star. Existing theories (see Dibay and Kaplan, 1976; Vainstein, 1983) give unambiguously a power spectrum of turbulent velocity $V(L) \propto L^{+*}$, with the spectral index $s$ depending on the physical conditions in the convection zone (from $s=1 / 2$ for Vajan convection with a strong magnetic field, to $s=1 / 4$ for acoustic turbulence). The resulting dependence is

$$
\nu\left(L>L_{0}\right) \propto \int N(L) \frac{V(L)}{L} d L \propto L^{-2+s} .
$$

Using $E(L)$ we obtain

$\nu\left(E>E_{0}\right) \propto \begin{cases}E_{0}^{-1+s / 2} \propto E_{0}^{-(0.88-0.75)} & \text { for rapidly decreasing magnetic field } \\ E_{0}^{-0.66+s / 3} \propto E_{0}^{-(0.58-0.5)} & \text { for slowly decreasing magnetic field }\end{cases}$

This theoretical consideration gives us not only the power-law character of the flare rate spectrum, but also indices that are similar to the observed ones. Observations of all nearby flaring red dwarfs, as well as solar flares in $\mathrm{H} \alpha$ and in microwaves, and of relatively older flare stars in Praesepe and Pleiades (see Gershberg and Shakhovskaya, 1984; Gershberg, 1988, 1989) comply with this theoretical result. However, the younger flare stars in the Orion cluster have spectra with steeper slopes than theoretically predicted.

\section{Possible astrophysical consequences}

The approach allows us to investigate turbulence in convection zones from the properties of the flare rate spectrum.

a) The dependence of flare rate spectrum upon activity phase. The long-term variations of the magnetic field during an activity cycle must influence the turbulence and thus also the flare rate spectrum. The ratio of the magnetic energy to the energy of the turbulent motions on different scales must be a basic parameter.

In this connection Kasinskij and Sotnikova (1990) and Gershberg (1990) have pointed out that the solar flare rate spectral index in the radiowave range changes during the 11-year solar activity cycle. for some of them. There are indications of changes in the flare rate spectrum index during several years for the classical flare star UV Ceti (Lacy et al., 1976).

b) Evolutionary changes in convection zones. There are possible systematic changes in the flare rate spectrum index with the age of flare stars, as detected by Korotin and Krasnobabtsev (1985); see also Gershberg (1989). If a flare rate spectrum reflects a turbulence velocity spectrum, then the deviating Orion results indicate possible momentary realizations of anomalous turbulence spectra, 
suggesting that the maximum occurs in small scales during early stages of stellar evolution. This phase is extremely transient $\left(\leq 10^{7}\right.$ years) and the spectrum changes quickly to that of Kolmogorov or Kraynovsky turbulence. In principle, the possibility of such exotic spectra is pointed out in a number of papers (Vainstein, 1983), though their realization is unlikely.

I sincerely thank Dr. R.E. Gershberg for his continuing support.

\section{References}

Dibai, E.A. and Kaplan, S.A., 1976, Dimensionality and Similarity of Astrophysical Quantities (in Russian), Nauka, Moscow, p. 224

Gershberg, R.E.: 1972, Astrophys. Space Sci. 19, 75

Gershberg, R.E., Pikel'ner, S.B.: 1972, Comments Astrophys. Space Sci. 4, 113

Gershberg, R.E., Shahovskaya, N.I.: 1984, Astrophys. Space Sci. 85, 235

Gershberg, R.E.: 1988, Mem. Soc. Astron. Ital. 59, 1

Gershberg, R.E.: 1989, Mem. Soc. Astron. Ital. 60, 263

Gershberg, R.E.: 1990, a private communication

Kasinskij, V.V., Sotnikova, R.T.: 1990, Issled. Geomagn. Aeron. Fiz. Solntsa, Moscow, No 83 , p. 99

Korotin, S.A., Krasnobabtsev, V.I.: 1985, Izv. Krym. Astrophys. Obs. 73, 131

Lacy, C.N., Moffet, T.J., Evans, D.S.: 1976, Astrophys. J. Suppl. Ser. 30, 85

Pustil'nik, L.A.: 1988, Sov. Astron. Lett. 14. 5, 398

Vainstein, S.A.: 1983, Magnetic Fields in the Space, Nauka, Moscow 